\title{
Regional cerebral blood flow velocity changes after indomethacin infusion in preterm infants
}

\author{
N C Austin, P W Pairaudeau, T K Hames, M A Hall
}

\begin{abstract}
Cerebral blood flow velocity was assessed during infusion of indomethacin over $\mathbf{3 0}$ minutes. Eleven preterm infants with symptomatic patent ductus arteriosus were studied on 12 occasions. Indomethacin infusion was associated with a significant reduction in time averaged mean velocity (TAMV), peak systolic velocity (PSV), and end diastolic velocity in both the anterior cerebral artery and middle cerebral artery. The fall in the TAMV and PSV was gradual with maximal change 30-40 minutes after the start of the infusion. It was concluded that administration of indomethacin by slow infusion produces haemodynamic alterations to the cerebral circulation comparable in magnitude with changes described with bolus administration. Indomethacin remains a useful and effective treatment for patent ductus arteriosus in preterm infants, but should continue to be used with caution.
\end{abstract}

Indomethacin is widely used in the treatment of symptomatic patent ductus arteriosus in preterm infants. The effect of indomethacin treatment on the cerebral circulation of the neonate has been assessed by Doppler ultrasound ${ }^{1-7}$ and near infrared spectroscopy ${ }^{8}$ in recent years. Most investigators ${ }^{1-6}$ have studied the effect of rapid (up to five minutes) injection of indomethacin on blood flow velocity in the anterior cerebral artery (ACA) or internal carotid artery (ICA). These studies have shown a rapid decline in mean blood flow velocity by up to $60-75 \%$ of pretreatment levels within five minutes of the completion of the injection. A decrease in both the peak systolic and diastolic velocities accounted for the fall in mean blood flow velocity. The findings of one study, ${ }^{7}$ using 'blind' isonation of the ACA with continuous wave Doppler, suggested that infusion of indomethacin over 20 minutes avoids the fall in cerebral blood flow velocity (CBFV) seen with more rapid administration. However, a more recent study using near infrared spectroscopy ${ }^{8}$ did not confirm these findings and failed to show a difference in the fall in total cerebral blood flow when injection of indomethacin over five minutes was compared with slow infusion (20-30 minutes).

To date there have been no studies of the effects of indomethacin on different regional areas of the cerebral circulation of human infants. In the following report both the anterior and the middle cerebral arteries were studied using duplex scanning, to determine whether prolonged infusion (over 30 minutes) of indomethacin was associated with changes in CBFV and if so whether regional differences in CBFV could be demonstrated.

\section{Method}

Eleven infants with echocardiographic confirmation of patent ductus arteriosus were selected for indomethacin treatment on clinical grounds. All cases had failed to respond to a trial of 48 hours of fluid restriction $(120 \mathrm{ml} / \mathrm{kg})$. One infant received two courses of indomethacin 21 days apart. Indomethacin was given via a peripheral vein in a dose of $0.2 \mathrm{mg} / \mathrm{kg} /$ dose 12 hourly for three doses. The dose was diluted in $1 \mathrm{ml}$ of sterile water and infused over 30 minutes. The drug infusion system was connected via a butterfly needle close to the cannula site in the vein to ensure complete drug administration over the 30 minutes. Only first dose studies for each course are reported.

Arterial or capillary carbon dioxide concentrations, blood pressure, and fluid intake were measured in the 24 hours before indomethacin administration. During the infusion heart rate, transcutaneous oxygen and carbon dioxide levels (if available), and blood pressure were monitored.

Two vessels were insonated using a Hewlett Packard duplex scanner with a $5 \mathrm{MHz}$ short focus probe and $100 \mathrm{~Hz}$ wall thump filter. Measurements were recorded on video tape for later analysis. The ACA was insonated, via the fontanelle, as it runs up toward the corpus callosum. The angle between the probe and artery could be visualised and the probe position was adjusted so that the angle of insonation at the time of study, before data collection, was less than or equal to 10 degrees for which no correction was made (a $3 \%$ error). The middle cerebral artery (MCA) was insonated transcranially through the temporal bone as the vessel leaves the circle of Willis and again the angle was maintained close to zero degrees in all cases. The depth of the probe from the skin surface was recorded and an attempt made to keep this constant; alternatively the closest strongest signal was used.

Echocardiographic assessment of left ventricle dimensions, left atrial to aortic ratio, and pulmonary artery and descending aorta Doppler velocities were performed before treatment and one hour later. 
Vasoscan (Oxford Sonocaid) was used to provide off line spectral analysis of the taped data. The parameters measured were peak systolic velocity (PSV), end diastolic velocity (EDV), and time averaged mean velocity (TAMV) calculated from the instantaneous mean velocity. Doppler examinations were carried out in the 10 minutes before infusion, at 5 minutes and at 10 minute intervals until 60 minutes, and in some babies up to 2 hours, after the start of indomethacin infusion. At least three segments of 3 to 9 seconds' duration were averaged to obtain the mean TAMV, PSV, and EDV.

The mean pretreatment value for TAMV, PSV, and EDV were compared with the maximum change or nadir in the fall observed and the change at 60 minutes. A paired $t$ test was used to detect a significant difference. Results are shown as mean (SD).

The coefficient of variation (SD as a percentage of the mean) was calculated for the pretreatment values for TAMV, PSV, and EDV for each infant; a percentage change greater than 2 SD from the group mean should be significant at the $95 \%$ confidence limits.

Six preterm infants, without echocardiographic evidence of a patent ductus arteriosus, were assessed for at least 30 minutes using a similar protocol and handling as in the study to assess whether any changes could be attributed to the study procedure.

Parental consent and ethics committee approval were obtained.

\section{Results}

The median birth weight was $1030 \mathrm{~g}$ (range 680-1850), median gestation at birth 27 weeks (range 24-32 weeks), and median postnatal age at treatment 9 days (7-30). Twelve studies in 11 infants were analysed. Eight babies were receiving artificial ventilation at the time of the first dose. The median arterial carbon dioxide tension pretreatment was $6 \cdot 1 \mathrm{kPa}$ (range $4 \cdot 1-7 \cdot 4$ ). The median total fluid intake in the 24 hours before treatment was $133.5 \mathrm{ml} / \mathrm{kg}$ (range 96-190) and the median haemoglobin concentration $118 \mathrm{~g} / \mathrm{l}$ (range 93-159). There was no correlation between the carbon dioxide level, fluid intake, or haemoglobin concentration and the changes in the TAMV, PSV, or EDV. Five of the infants had continuous blood pressure monitoring and transcutaneous measurement of oxygen and carbon dioxide. No significant changes were recorded. In five others the measurements were intermittent.

An average of 4.25 segments of the scan, each of 6.7 seconds' duration or an average of

\begin{tabular}{lllllll} 
Table 1 & Statistical analysis of the TAMV & & & \\
\hline Site & Time & $\begin{array}{l}\text { Mean velocity } \\
\text { change }\end{array}$ & & $\begin{array}{l}95 \% \text { Confidence } \\
\text { interval }(\mathrm{cm} / \mathrm{s})\end{array}$ & p Value \\
\cline { 3 - 5 } $\mathrm{cm} / \mathrm{s}$ & $\%$ & & & \\
\hline MCA & Maximum change & 4.36 & -40.0 & 2.91 to 5.817 & $<0.001$ \\
& 60 minutes & 3.65 & -31.6 & 2.03 to 5.26 & $<0.001$ \\
ACA & Maximum change & 2.70 & -32.9 & 1.67 to 3.74 & $<0.001$ \\
& 60 minutes & 1.98 & -24.0 & 0.72 to 3.24 & $<0.01$ \\
\hline
\end{tabular}

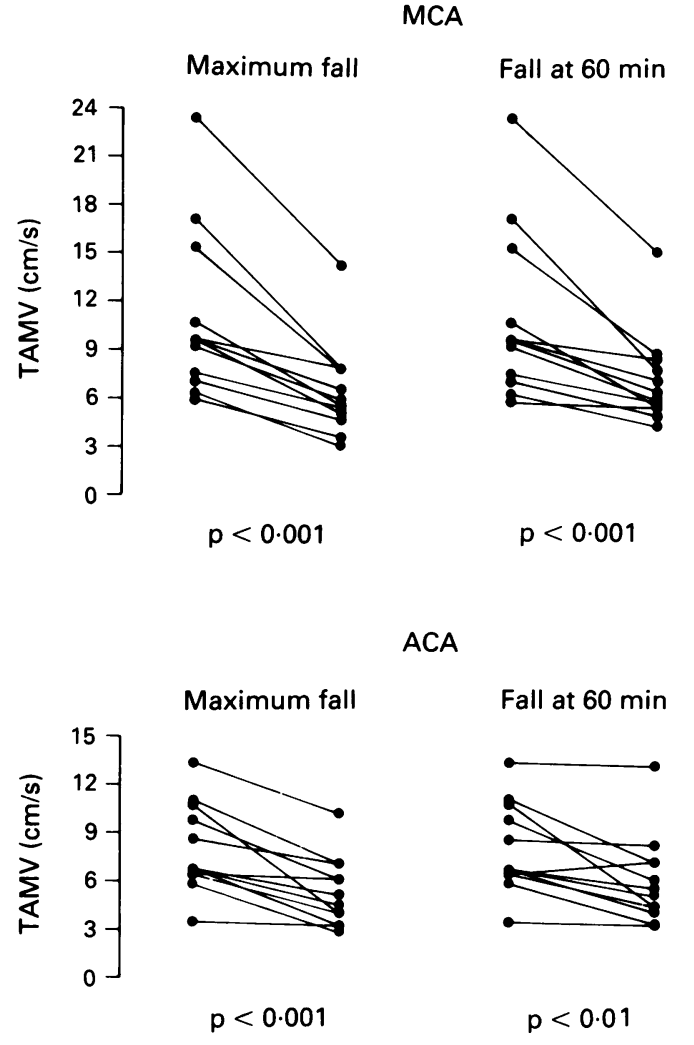

Figure 1 Change in the TAMV after indomethacin infusion.

70 cardiac cycles, were analysed per measurement.

Table 1 shows that the maximum fall in TAMV was $-40.0 \%$ in the MCA and $-32.9 \%$ in the ACA; this occurred at 36.5 and 31.5 minutes (median) after the start of indomethacin infusion respectively. These values were compared with pretreatment recordings to determine whether a significant change had occurred. The absolute changes in the TAMV for each artery are shown at maximum fall and at 60 minutes in figure 1 . The maximum fall was significant $(p<0.001)$ in both vessels and was more pronounced in the MCA $(p<0.001)$ than the ACA $(p<0.01)$ at 60 minutes.

The maximum fall in the PSV was $-30.6 \%$ in the MCA and $-27.0 \%$ in the ACA and occurred at 32.5 and 31.5 minutes (median) respectively (table 2 ). The absolute changes in the PSV for each artery are shown at maximum fall and at 60 minutes from the start of infusion in figure 2 . The maximum fall and fall at 60 minutes were significant $(p<0.001)$ for both arteries.

The maximum fall in the EDV was $-75 \cdot 5 \%$ in the MCA and $-58 \cdot 1 \%$ in the ACA and occurred at 22 and 17 minutes respectively (median). This was highly significant $(p<0.001)$ in both vessels, and remained significant in the MCA at 60 minutes $(p<0.05)$ but not in the ACA (table 3$)$.

The difference in the magnitude of the fall between the MCA and ACA was significant for the TAMV at maximum fall $(p<0.05)$, and for the PSV at maximum fall $(p<0.01)$ and at 60 minutes $(p<0.05)$, reflecting the higher 
Table 2 Statistical analysis of the PSV

\begin{tabular}{|c|c|c|c|c|c|}
\hline \multirow[t]{2}{*}{$\overline{\text { Site }}$} & \multirow[t]{2}{*}{ Time } & \multicolumn{2}{|c|}{$\begin{array}{l}\text { Mean velocity } \\
\text { change }\end{array}$} & \multirow[t]{2}{*}{$\begin{array}{l}95 \% \text { Confidence } \\
\text { interval }(\mathrm{cm} / \mathrm{s})\end{array}$} & \multirow[t]{2}{*}{ p Value } \\
\hline & & $\mathrm{cm} / \mathrm{s}$ & $\%$ & & \\
\hline \multirow[t]{2}{*}{$\overline{\mathrm{MCA}}$} & Maximum change & $15 \cdot 71$ & $-30 \cdot 6$ & 12.36 to 19.05 & $<0.001$ \\
\hline & 60 minutes & $13 \cdot 85$ & $-25 \cdot 3$ & 9.65 to 18.05 & $<0.001$ \\
\hline \multirow[t]{2}{*}{ ACA } & Maximum change & $9 \cdot 28$ & $-27 \cdot 0$ & 6.74 to 11.83 & $<0.001$ \\
\hline & 60 minutes & $7 \cdot 39$ & $-21 \cdot 0$ & 4.37 to 10.41 & $<0.01$ \\
\hline
\end{tabular}

Table 3 Statistical analysis of the EDV

\begin{tabular}{lllllll}
\hline Site & Time & \multicolumn{2}{l}{$\begin{array}{l}\text { Mean velocity } \\
\text { change }\end{array}$} & & $\begin{array}{l}\text { 95\% Confidence } \\
\text { interval }(\mathrm{cm} / \mathrm{s})\end{array}$ & p Value \\
\cline { 3 - 4 } $\mathrm{cm} / \mathrm{s}$ & $\%$ & & & \\
\hline MCA & Maximum change & 3.11 & -75.5 & 1.66 to 4.56 & $<0.001$ \\
& 60 minutes & 1.81 & -44.0 & 0.47 to 3.16 & $<0.05$ \\
ACA & Maximum change & 2.00 & -58.1 & 1.02 to 2.99 & $<0.001$ \\
& 60 minutes & 0.76 & -22.1 & -0.04 to 1.57 & $\mathrm{NS}$ \\
\hline
\end{tabular}

pretreatment velocities in the MCA. There was no significant difference in the diastolic velocity changes between the vessels. A trend was observed of a greater percentage fall in the TAMV and PSV in the MCA compared with the ACA, but this did not reach significance.

The resistance index (PSV-EDV/PSV) was calculated for both vessels. Pretreatment values were $0.93(0.05)$ in the MCA and 0.91 $(0.05)$ in the ACA. The resistance index increased significantly in both vessels to 0.97 $(0.04) \quad(p<0.01)$ and $0.95 \quad(0.05) \quad(p<0.05)$ respectively at maximum fall. At 60 minutes the resistance index was still significantly increased in the MCA $0.95(0.04)(p<0.05)$,
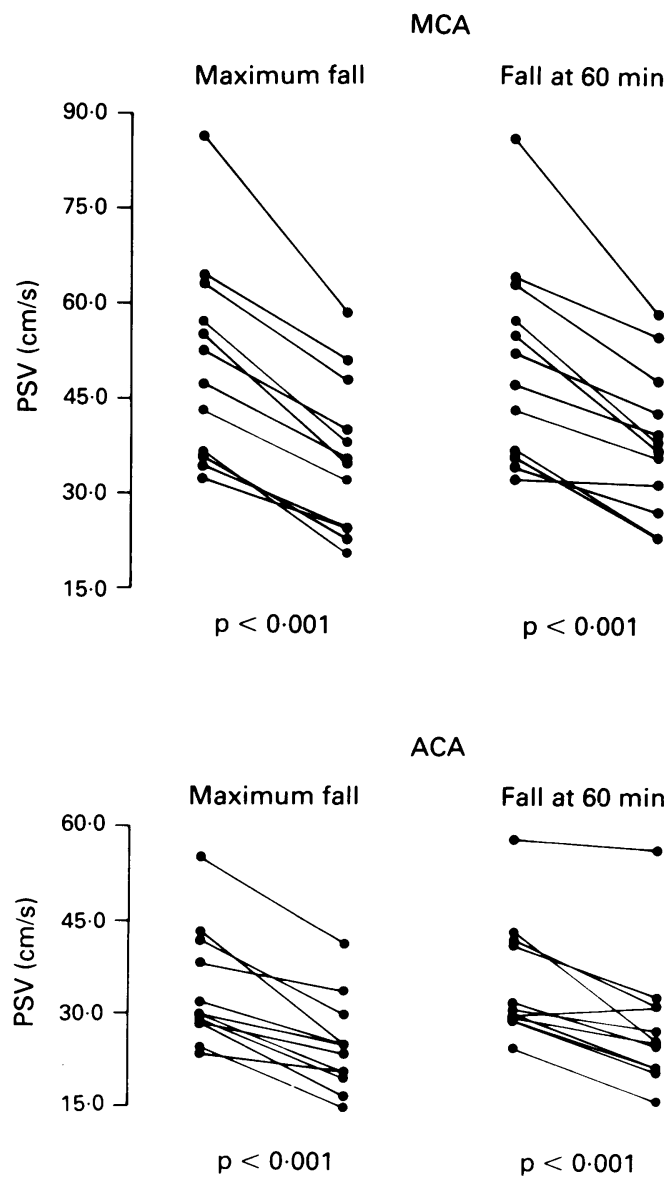

Figure 2 Change in the PSV after indomethacin infusion. but had returned to pretreatment levels in the ACA.

The coefficient of variation for the TAMV measurements of the group before indomethacin was $6.0 \%(2.6)$, and for the PSV was $4 \cdot 8 \%(2 \cdot 2)$, so that a change of greater than $12 \%$ and $10 \%$ respectively would be significant at the $95 \%$ confidence interval. These values did not alter significantly at maximum fall or 60 minutes. The coefficient of variation for the EDV was $21.1 \%(16.9)$, reflecting the effect of small changes on already very low diastolic velocities seen in the presence of patent ductus arteriosus.

In the six untreated infants studied serially, the coefficient of variation for the TAMV measurements throughout the 30 minutes was $7 \cdot 2(6 \cdot 1) \%$; for the PSV measurements it was $5.4(3.2) \%$; and for the EDV $15.4(11 \cdot 2) \%$. These are not statistically different from the pretreatment values for variability in the 11 study infants and provide evidence that there were no consistent changes induced by the study procedure.

In all infants the echocardiographic assessment at 60 minutes still showed abnormal Doppler patterns in the pulmonary artery consistent with left to right ductal shunting, with no significant change in the $M$ mode left ventricle dimensions and left atrial to aortic ratio (pretreatment $1.57(0.28), 60$ minutes $1.56(0.24))$.

The efficacy of indomethacin in closure of the ductus did not appear to be altered by slow infusion. Ductal closure occurred in seven. One infant required surgical ligation after two courses failed to produce sustained closure of the ductus. In another infant ductal closure occurred after the second course, while two others had significant reduction in the ductal flow but without loss of the ductal murmur; on follow up one has subsequently closed and the other is awaiting surgery.

\section{Discussion}

Our study is methodologically different from previous studies in several aspects. First, duplex scanning (which employs pulsed Doppler) allows visualisation and correction for the angle between the probe and artery (if the angle is not close to zero degrees), while the continuous wave Doppler methods used in previous studies' 467 are 'blind' and assume a constant angle between the probe and artery and constant site. This may result in errors in an intermittent study. ${ }^{9}$ Second, we have reported the changes in all parameters, TAMV, PSV, and EDV, along with coefficient of variation data and used a larger number of velocity waveforms in their calculation. ${ }^{10}$ Third, the ACA was insonated nearer its origin where it is of larger diameter and at a comparable site despite intermittent technique. Fourth, we have reported only first dose studies as the combination of first and repeated doses may be misleading as two studies $^{1} 4$ showed no significant fall with repeated bolus doses of indomethacin. 
Finally, we have used larger numbers of infants for statistical analysis than have been used in previous studies.

Our results show that slow infusion of indomethacin was associated with a significant reduction in the cerebral blood flow velocity parameters of TAMV, PSV, and EDV at maximum change as measured by duplex scanning. The changes were sustained to 60 minutes for the TAMV and PSV in both vessels studied. The changes in TAMV at one hour $(-31.6 \%$ (MCA) and $-24 \%$ (ACA)) are of a similar order to those of previous reports using rapid infusion of indomethacin where falls in mean velocity of $28 \% \%^{4}$ and $20 \%{ }^{7}$ were seen in studies using continuous wave Doppler and $38 \%,{ }^{2} 35 \%,{ }^{3} 36 \%{ }^{5}$ in those using duplex scanning. In our study the maximum fall usually occurred by $30-40$ minutes for both TAMV and PSV. This differs from the abrupt fall in mean velocity measurements in the first five minutes seen after rapid administration of indomethacin, 167 which we have also seen in earlier studies in our unit.

In the MCA, the TAMV and PSV changes were greater in magnitude, tended to have a greater percentage change, and the EDV a more prolonged change, than those in the ACA. No other study has compared the response in different cerebral vessels despite documented differences in Doppler parameters between the cerebral vessels under normal conditions. ${ }^{11-14}$ It appears from our results that the effects of indomethacin on the EDV were more pronounced on the MCA than the ACA.

Diastolic velocity is dependent on the systemic blood pressure, carbon dioxide tension, vascular resistance, intracranial pressure and, in the presence of a ductus, the degree of ductal steal. 91315 In our study the echocardiographic assessment at 60 minutes, which showed persistent patent ductus arteriosus with no significant change in the $M$ mode parameters, suggests that changes in the diastolic velocity are drug related rather than ductus related and they are in the opposite direction to that seen with a closing ductus. ${ }^{13}$ The pattern of change to the diastolic velocity observed in this study in both vessels would be in keeping with the hypothesis that indomethacin has a direct effect on cerebral blood vessels, causing a rapid constriction and increase in vascular resistance. Whether this is mediated by prostaglandin inhibition is still unknown. The more prolonged effect on the
EDV in the MCA also contributed to the continued highly significant fall in TAMV in that vessel at 60 minutes. These findings suggest that there may be regional differences in the response of preterm infants' cerebral vessels to indomethacin. Conclusions based on Doppler studies of the ACA alone could underestimate the effect on CBFV to other areas of the brain.

Our findings show that slow infusion of indomethacin causes a significant reduction in TAMV, PSV, and EDV in both the ACA and MCA. The fall in these values is more gradual than with rapid infusions. Indomethacin remains a useful and effective treatment for patent ductus arteriosus in preterm infants, but should be used with caution as we conclude that administration by slow infusion does produce haemodynamic alterations to the cerebral circulation.

Dr NC Austin was supported by The Jules Thorn Charitable Trust.

Cowan F. Indomethacin, patent ductus arteriosus, and cerebral blood flow. $₹$ Pediatr 1986;109:341-4.

2 Lundell BPW, Sonesson S-E, Cotton RB. Ductus closure in preterm infants. Acta Paediatr Scand [Suppl] 1986; 329:140-7.

3 Evans DH, Levene MI, Archer LNJ. The effect of indomethacin on cerebral blood flow velocity in premature infants. Dev Med Child Neurol 1987;29:776-82.

4 Laudignon N, Chemtob S, Bard H, Aranda JV. Effect of indomethacin on cerebral blood flow velocity of premature newborns. Biol Neonate 1988;54:254-62.

5 Lundell BPW, Sonesson S-E, Sundell H. Cerebral blood flow following indomethacin administration. Dev Pharmacol Ther 1989;13:139-44.

6 Van Bel F, Van de Bor M, Stijnen T, Baan J, Ruys JH. Cerebral blood flow velocity changes in preterm infants arter a single dose of indomethacin: duration of its effect. Pediatrics 1989;84:802-7.

7 Colditz P, Murphy D, Rolfe P, Wilkinson AR. Effect of infusion rate of indomethacin on cerebrovascular infusion rate of indomethacin on cerebrovascular responses

8 Edwards $\mathrm{AD}$, Wyatt JS, Richardson $\mathrm{C}$, et al. Effects of indomethacin on cerebral haemodynamics in very preterm infants. Lancet 1990;335:1491-5.

9 Winkler P, Helmke K. Major pitfalls in Doppler investigations with particular reference to the cerebral vascular system. Pediatr Radiol 1990;20:219-28.

10 Anthony MY, Evans DH, Levene MI. Cyclical variations in cerebral blood flow velocity. Arch Dis Child 1991; 66: $12-6$.

11 Evans DH, Levene MI, Shortland DB, Archer LN. Resistance index, blood flow velocity, and resistance area product in the cerebral arteries of very low birth weight infants during the first week of life.Ultrasound Med Biol 1988;14:103-10.

12 Horgan JG Rumack CM, Hay $T$, et al. Absolute intracranial blood flow velocities evaluated by duplex intracranial blood flow velocities evaluated by duplex Doppler sonography in asymptom

13 neonates. AFR 1989;152:1059-64. Wright LL, Baker KR, Hollander DI, et al. Cerebral blood flow velocity in term newborn infants: changes

14 Raju NK, Zikos E. Regional cerebral blood velocity in infants. F Ultrasound Med 1987;6:498-507.

15 Levene MI, Shortland D, Gibson N, Evans DH. Carbon dioxide reactivity of the cerebral circulation in extremely premature infants: effects of postnatal age and indomethacin. Pediatr Res 1988;24:175-9. 